\title{
Management of Large Bowel Injury During Laparoscopic Surgery
}

\author{
Hegde C. V.
}

It is a given that the bowel has to be adequately prepared prior to every laparoscopic surgery as an insurance against fecal contamination in the event of an injury. The large bowel in general, and the sigmoid colon in particular, runs a risk of iatrogenic injury in cases of severe endometriosis, pelvic inflammatory disease, previous exploration for myomectomy, or in cases of previous laparoscopic surgery resulting in the formation of dense adhesions.

In a series of 2,084 patients who underwent a laparoscopic-assisted type I vaginal hysterectomy, six patients had large bowel injuries [1].

The question that stares us in the face is whether sigmoid colon injury during laparoscopic surgery requires repair by exploratory laparotomy or whether a laparoscopic repair would suffice. There is sometimes a touching belief of some laparoscopic surgeons not trained otherwise in bowel surgery that they can get away by mere approximation of the cut edges of an open bowel. This is a dangerous ostrich-like head in the sand approach. Seromuscular defects that do not expose the intestinal submucosa do not require repair. However, it would be prudent and wise to approximate such defects using synthetic 3-0 absorbable sutures. Transmural defects through which the intestinal submucosa or mucosa is visible, require repair.

Hegde C. V. ( $₫)$, Professor

Department of Obstetrics and Gynecology, T.N. Medical

College, Mumbai, India

e-mail: dr.c.v.hegde@gmail.com
Conventionally, colorectal surgeons advise a two-layer closure approach-the first, a full thickness continuous stitch running perpendicular to the length of the bowel and the second, an interrupted seromuscular stitch to cover the first layer as further protection. The edges of the damaged bowel may need "freshening" [2]. The first layer may be of 3-0 synthetic absorbable material. Bowel integrity needs to be checked at this juncture and this can be done by checking for escape of air bubbles on pressing or milking the proximal part of the repaired bowel while occluding the distal part, all the while insuring the sutured site remains submerged in saline. At laparoscopy, air can be instilled per rectum to check for leakage. Upon the confirmation of an absence of leakage, the second layer of 4-0 silk may be placed. A single layer inverting interrupted closure is acceptable for defects less than $5 \mathrm{~mm}$ where the sutures are placed at $2-3-\mathrm{mm}$ intervals and 1-2 $\mathrm{mm}$ from the edge of the defect.

Therefore, it is mandatory that any primary repair done laparoscopically needs to be meticulously done. Only then, in the now emerging trend of primary closure of large bowel injury in the absence of significant bowel contamination, will this method be deemed safe. A protective colostomy may not be performed in these circumstances. End colostomy, however, may still be necessary when there is severe fecal contamination, shock, severe blood loss of more than $1,000 \mathrm{ml}$, or in cases of a delayed diagnosis of perforation as happens in coagulation injury at laparoscopy [3].

Regarding colostomy, the procedure by itself does not guarantee the prevention of an anastomotic leak. Several 
prospective studies have examined the role of fecal diversion in colon trauma; these have been succinctly summarized by a Cochrane meta-analysis from 2009. Comprised of six studies with 705 patients, this analysis confirms no difference in mortality between patients treated with fecal diversion or primary repair. Additionally, primary repair results in substantially less morbidity, with lower rates of total complications (OR 0.54), intra-abdominal infections (OR 0.67), total infections (OR 0.44), and wound complications (OR 0.73) relative to colostomy. Similarly, Maxwell and Fabian compiled data from 20 retrospective studies and found an overall complication rate of $14 \%$ for primary repair versus $31 \%$ for colostomy, with equivalent mortality [4].

According to Reich et al. [5], if the large bowel is involved, the treatment options include primary repair, colostomy, or segmental resection. Resection is mandatory in thermal injuries. Intraoperative lacerations may be repaired laparoscopically according to the size of the lesion, surgeon experience, and preoperative bowel preparation.

Proctosigmoidoscopy can be performed at the end of the surgery to evaluate intraluminal abnormality or rectosigmoid injury. The pelvis is then filled with isotonic fluid and observed laparoscopically for air leakage.

The use of drains almost brooks no debate and indeed may be considered mandatory. However, its routine use may be contentious. When used, it is commonly removed after 5 days. However, several prospective randomized trials have demonstrated that there is no significant difference in postoperative morbidity and mortality between patients who do and those who do not undergo closed-suction drainage [6-8]. There are no significant differences in anastomotic leaks (clinical or radiologic) or infectious morbidities. I do suspect, however, it would take a surgeon with nerves of steel to not place a drain under these circumstances.

Postoperatively, it is prudent to start fluids early rather than late. This flies in the face of the "conventional" practice of keeping patients starving arbitrarily for 5 days. The secretion of intestinal fluids is unabated and the patient is never really "nil by mouth." With the advancement of gastrointestinal laparoscopy, surgeons have moved away from the traditional practice of postoperative nasogastric decompression and oral feeding only after passage of flatus has signaled the resumption of bowel function. Several prospective randomized trials have demonstrated that early feeding or patient-controlled feeding is safe in patients undergoing both laparoscopic and open bowel resections and anastomosis [9-13]. Furthermore, nasogastric decompression was discontinued immediately after the surgery in the majority of these studies. Postoperative nasogastric tube decompression does not provide any significant benefit in gynecology patients, including those undergoing bowel resection [14]. Although the surgeon should make decisions based on each specific case, it is reasonable to begin clear liquids as early as the first postoperative day. When bowel function resumes or an adequate volume of the clear liquid diet is consumed, a regular or low-residue solid diet may be instituted. Standard postoperative intravenous fluids should be maintained until the patient demonstrates an adequate oral intake.

Early recognition of a failed repair or anastomosis is vital to patient outcome. Leaks present as peritonitis, an intra-abdominal abscess, fistula, and sepsis. A majority of leaks occur within 5-7 days of the original surgery. In a recent study of 655 consecutive patients undergoing colonic and rectal anastomoses, the authors report a $6 \%$ rate of clinical leaks [15]. If there is radiologic evidence of a leak or a strong clinical suspicion, immediate re-exploration is warranted with repair of the failure/leak site and proximal diversion by colostomy.

Prevention of large bowel injury at laparoscopy includes techniques like gentle bowel manipulation, the use of sharp tissue dissection, dissection of tissue from non-adherent areas to the adherent sites, bowel sparing tissue dissection, a meticulous use of electrosurgery, etc. However, at the end of surgery, a search to detect any undetected bowel injury if any is mandatory.

Laparoscopic management of cases of severe endometriosis or cases where dense pelvic adhesions are suspected includes apprising the patient of the possibility of bowel injury during such surgery and providing her the confidence about such an event being dealt with competently if the need arose. The drill that the surgeon needs to follow is exacting which entails a detailed preoperative clinical and radiologic assessment, meticulous bowel preparation, a mental visualization of the surgical process, and an involvement of a surgeon experienced in management of large bowel injury. Procrastination can mean that a generally forgiving organ that responds well to insult and injury if treated well and in time may later turn its back with fatal consequences.

\section{References}

1. Shen $\mathrm{C}, \mathrm{Lu} \mathrm{H}$, Chang S. Characteristics and management of large bowel injury in laparoscopic-assisted vaginal hysterectomy. J Am Assoc Gynecol Laparosc. 2002;9:35-9.

2. Sweeny KJ, Joyce M, Geraghty JG. Management of intraoperative bowel injuries. CME J Gynecol Oncol. 2002;7:178-82.

3. Gonzalez RP, Merlotti GL, Holevar MR. Colostomy in penetrating colon injury: is it necessary? J Trauma. 1996;41:271-5.

4. Maxwell RA, Fabian TC. Current management of colon trauma. World J Surg. 2003;27:632-9.

5. Reich H, McGlynn F, Budin R. Laparoscopic repair of fullthickness bowel injury. J Laparoendosc Surg. 1991;1:119-22. 
6. Sagar P, Hartley M, Macfie J, et al. Randomized trial of pelvic drainage after rectal resection. Dis Colon Rectum. 1995;38: 254-8.

7. Sagar P, Couse N, Kerin M, et al. Randomized trial of drainage of colorectal anastomosis. Br J Surg. 1993;80:769-71.

8. Urbach D, Kennedy E, Cohen M. Colon and rectal anastomoses do not require routine drainage. Ann Surg. 1999;229:174-80.

9. Reissman P, Teoh T, Cohen S, et al. Is early oral feeding safe after elective colorectal surgery? A prospective randomized trial. Ann Surg. 1995;222:73-7.

10. Hartsell P, Frazee R, Harrison J, et al. Early postoperative feeding after elective colorectal surgery. Arch Surg. 1997;132:518-20.

11. Pearl M, Valea F, Fischer M, et al. A randomized controlled trial of early postoperative feeding in gynecologic oncology patients undergoing intra-abdominal surgery. Obstet Gynecol. 1998;92: 94-7.
12. Behrus K, Kircher A, Galanko J, et al. Prospective randomized trial of early initiation and hospital discharge on a liquid diet following elective intestinal surgery. J Gastrointest Surg. 2000;4: 217-21.

13. Han-Geurts I, Jeekel J, Tilanus H, et al. Randomized clinical trial of patient controlled versus fixed regimen feeding after elective abdominal surgery. Br J Surg. 2001;88:1578-82.

14. Pearl M, Valea F, Fischer M, et al. A randomized controlled trial of postoperative nasogastric tube decompression in gynecologic oncology patients undergoing intraabdominal surgery. Obstet Gynecol. 1996;88:399-402.

15. Alves A, Panis Y, Pocard M, et al. Management of anastomotic leakage after nondiverted large bowel resection. J Am Coll Surg. 1999;189:554-9. 